06,11

\title{
Эволюция доменной структуры и формирование заряженных доменных стенок при переключении поляризации в монокристаллах ниобата лития, модифицированных отжигом в вакууме
}

\author{
(С) В.И. Пряхина, Д.О. Аликин, С.А. Негашев, В.Я. Шур \\ Институт естественных наук и математики, Уральский федеральный университет, \\ Екатеринбург, Россия \\ E-mail: viktoria.pryahina@urfu.ru
}

(Поступила в Редакцию 31 мая 2017 г.)

\begin{abstract}
Исследована эволюция доменной структуры при переключении поляризации в пластинах ниобата лития с пространственно неоднородной электропроводностью, созданной отжигом в вакууме. Изучено формирование заряженных доменных стенок в объеме. Обнаруженные особенности роста доменов в объеме отнесены за счет формирования нанодоменов в пироэлектрическом поле и неоднородного пространственного распределения электрического поля. Создание заряженных доменных стенок с контролируемыми параметрами представляет значительный интерес для инженерии доменных стенок.
\end{abstract}

Использовалось оборудование Уральского центра коллективного пользования „Современные нанотехнологии“ ИЕНиМ УрФУ.

DOI: 10.21883/FTT.2018.01.45295.178

\section{1. Введение}

Монокристаллы ниобата лития $\mathrm{LiNbO}_{3}$ (LN) являются наиболее популярным материалом для создания электрооптических и нелинейно-оптических устройств, поскольку LN обладает рекордными значениями нелинейно-оптических и электрооптических коэффициентов [1-3].

Доменная инженерия (domain engineering) - создание стабильной доменной структуры с заданными параметрами в сегнетоэлектриках, в настоящее время успешно используется для улучшения свойств кристаллов и изготовления функциональных устройств с улучшенными параметрами [4-7]. Кристаллы семейства LN являются наиболее популярными материалами для доменной инженерии. Кристаллы LN с прецизионной регулярной доменной структурой используются для преобразования длины волны лазерного излучения (генерации второй гармоники и параметрической генерации света) [4-6]. Одним из самых распространенных способов создания регулярной доменной структуры является переключение поляризации приложением пространственно неоднородного электрического поля с помощью системы полосовых электродов [6]. Создание нанодоменных структур с высокой концентрацией нейтральных и заряженных доменных стенок (ЗДС), так называемая инженерия доменных стенок (engineering of domain boundaries), позволяет существенно улучшать пьезоэлектрические свойства кристаллов [8-10]. Следует отметить, что в LN разработаны методики формирования квазирегулярных нанодоменных структур в сильно неравновесных условиях переключения [4-6]. Сравнительно простая доменная структура и возможность визуализировать домены на поверхности и в объеме различными методами с высоким пространственным разрешением $[11,12]$ позволяет использовать LN в качестве модельного материала для изучения кинетики самоорганизованного формирования нанодоменных структур и ЗДС [1,4-8].

Известно, что температурный отжиг пластин LN в восстановительной атмосфере используется для уменьшения пироэлектрического эффекта [13-15]. Так, отжиг в атмосфере с пониженным парциальным давлением кислорода (в частности, в вакууме) приводит к увеличению оптического поглощения в видимом и инфракрасном диапазоне $[1,13]$ и увеличению электропроводности [16,17]. Обычно используемый длительный отжиг приводит к однородному изменению свойств в пластине сегнетоэлектрика [13], однако уменьшение температуры и длительности отжига позволяет создавать поверхностные слои с повышенной электропроводностью $[15,17,18]$.

Существенное увеличение оптического поглощения в результате отжига не позволяет использовать отожженный „черный LN“ для оптических применений, поэтому переключение поляризации в модифицированных кристаллах ранее не исследовалось. Вместе с тем, эффекты отжига в восстановительной атмосфере в оксидах, как правило, обратимы. Высокотемпературный отжиг в кислородной среде позволяет восстановить исходные параметры LN [17]. Подобную операцию можно рассматривать, как возможность управлять ростом доменов за счет неоднородной модификации проводимости кристаллов.

В работе изучалась эволюция доменной структуры при переключении поляризации в кристаллах LN c пространственно неоднородной электропроводностью, созданной высокотемпературным отжигом в вакууме. Показано, что отжиг при температурах выше $800^{\circ} \mathrm{C}$, позволяет создавать неоднородное распределение переключающего поля и реализовать „внутриобъемное переключение“ - зарождение и рост доменов с ЗДС 


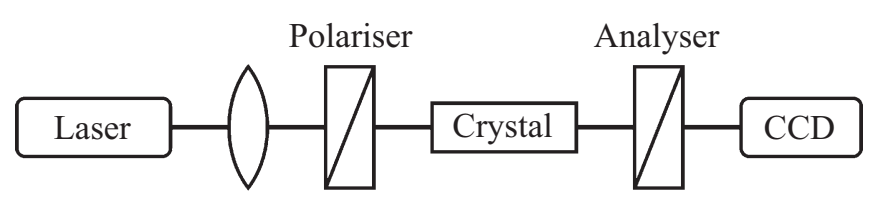

Рис. 1. Схема установки для измерения распределения электрического поля.

в объеме кристалла. Предложена модель эволюции доменной структуры в модифицированных кристаллах.

\section{2. Эксперимент}

Исследовались пластины конгруэнтного ниобата лития (CLN) (Crystal Technology, США) толщиной $0.5 \mathrm{~mm}$, вырезанные перпендикулярно полярной оси. Кристаллы CLN были выращены по методу Чохральского вытягиванием в направлении полярной оси.

Исследуемые образцы размерами $15 \times 10 \mathrm{~mm}$ отжигали в вакууме при давлении $10^{-5}$ Torr. Скорость нагрева до заданной температуры (табл. 1) и охлаждения после завершения отжига не превышала $1^{\circ} \mathrm{C} / \mathrm{s}$.

Для изучения эволюции доменной структуры при переключении поляризации использовалась экспериментальная установка на основе поляризационного оптического микроскопа [19], позволяющая регистрировать последовательность мгновенных доменных конфигураций.

Визуализация статической доменной структуры на полярной поверхности производилась при помощи: оптической микроскопии (Olympus BX61, Olympus, Япония), силовой микроскопии пьезоэлектрического отклика (CMПO) (зондовая нанолаборатория NTEGRA Aura, НТ-МДТ, Россия) и конфокальной микроскопии комбинационного рассеяния света (КМКР) (Alpha 300 AR, WiTec, Германия). СМПО позволяет визуализировать домены на поверхности с пространственным разрешением до $10 \mathrm{~nm}[11,20]$, а КМКР - в объеме с пространственным разрешением около $300 \mathrm{~nm}$ и разрешением по глубине $500 \mathrm{~nm}[11,12,21]$.

Домены на неполярной Ү-поверхности визуализировались при помощи оптической микроскопии после кратковременного селективного химического травления в плавиковой кислоте (HF) [22]. Время травления $2.5 \mathrm{~min}$.

Для измерения неоднородного распределения электрического поля использовалась оптическая схема, основанная на интерференции обыкновенного и необыкновенного лучей $[23,24]$. Схема установки приведена на рис. 1. Широкий линейно поляризованный луч лазера с длиной волны $633 \mathrm{~nm}$ проходил через образец вдоль

Параметры отжига образцов в вакууме

\begin{tabular}{c|c|c|c|c|c|c|c|c}
\hline Температура, ${ }^{\circ} \mathrm{C}$ & \multicolumn{2}{|c|}{300} & 800 & \multicolumn{4}{c}{850} \\
\hline Время отжига, min & 60 & 600 & 10 & 2 & 5 & 10 & 15 & 20
\end{tabular}

Y-оси кристалла. Угол между противоположными плоскостями (гранями) XZ исследуемого образца составлял $3^{\circ}$. Для фильтрации света, деполяризованного на неоднородностях кристалла, использовался анализатор. Интерференционная картина регистрировалась видеокамерой. Для приложения напряжения вдоль полярной оси на полярные поверхности образца наносились электроды из серебряной пасты. Пространственно неоднородное электрическое поле за счет линейного электрооптического эффекта приводило к сдвигу полос и изменению их формы.

Измерение поверхностной электропроводности осуществлялось двухзондовым методом при помощи пикоамперметра (Keithley 6485, США). Для измерения распределения электропроводности в объеме толщину образца последовательно уменьшали, удаляя слои толщиной от 10 до $100 \mu \mathrm{m}$ при помощи прецизионного шлифовально-полировального станка (PM5, Logitech Ltd., Великобритания), после чего измеряли поверхностную электропроводность.

\section{3. Распределение электропроводности и электрического поля в объеме}

Измерения показали, что электропроводность в исходных образцах CLN менее $10^{-14} \mathrm{~S}$. Отжиг в вакууме

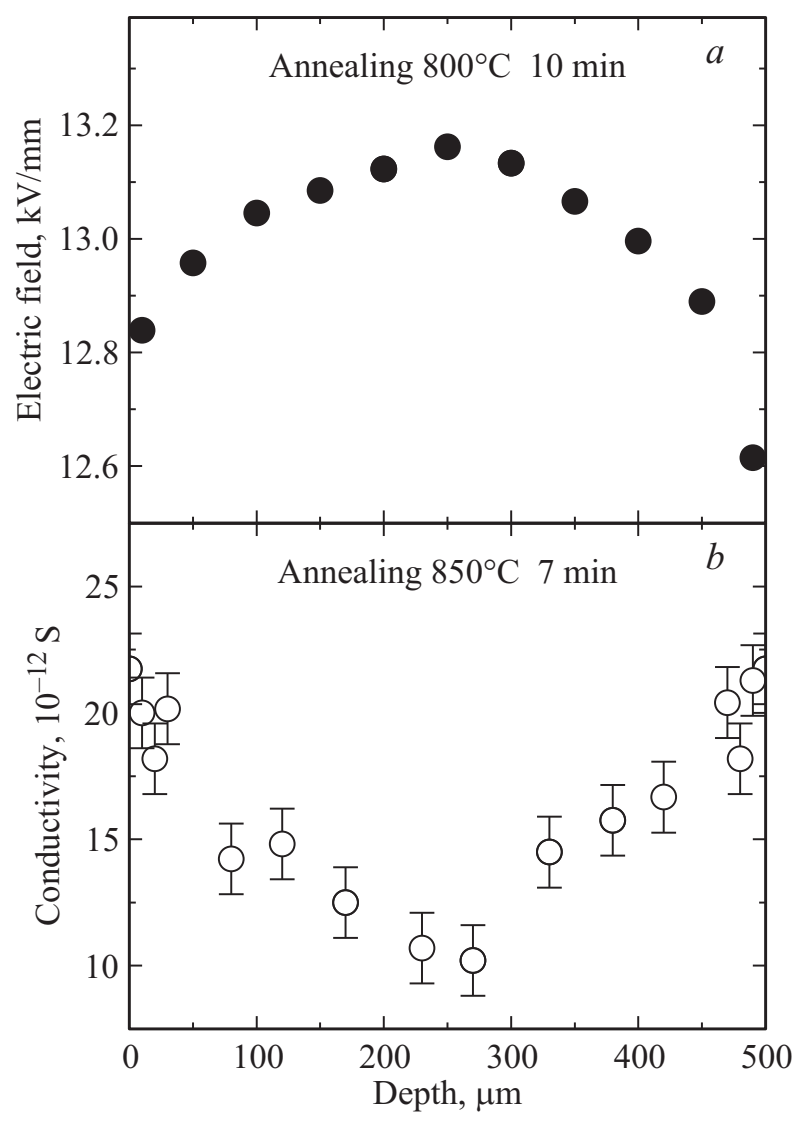

Рис. 2. Распределение по глубине $(a)$ электрического поля и $(b)$ электропроводности в отожженном CLN. 
при $300^{\circ} \mathrm{C}$ в течение $10 \mathrm{~h}$ не приводил к заметному изменению электропроводности и распределение электрического поля оставалось однородным.

Отжиг при $850^{\circ} \mathrm{C}$ длительностью более двух минут приводил к значительному увеличению поверхностной проводимости (до $10^{-12}-10^{-11} \mathrm{~S}$ ). При этом электропроводность в объеме кристалла была существенно меньше, чем вблизи поверхности, что приводило к неоднородному распределению электрического поля (рис. 2). Следует отметить, что процесс переключения в ниобате лилия определяется величиной превышения поля над пороговым значением $\left(E_{t h}\right) \Delta E=E_{e x}-E_{t h}$ [25], поэтому в полях близких к пороговому значению даже незначительное уменьшение поля вблизи поверхности приводит к переключению только в объеме.

\section{4. Пороговые поля переключения поляризации}

Для определения порогового поля переключения поляризации $E_{t h}$ регистрировалась эволюция доменной структуры в линейно растущем поле, и определялась величина поля, при которой появлялся первый оптически различимый домен. Увеличение длительности отжига при температуре $850^{\circ} \mathrm{C}$ приводило к пропорциональному понижению $E_{t h}$ (рис. 3). Значительное уменьшение величины порогового поля наблюдалось нами ранее в кристаллах CLN после ионно-плазменного облучения $[24,26]$.

\section{5. Влияние низкотемпературного отжига на эволюцию доменной структуры}

Отжиг в вакууме при низкой температуре $\left(300^{\circ} \mathrm{C}\right)$ незначительно изменял эволюцию доменной структуры. После отжига, в отличие от не отожженных кристаллов, при достижении внешним полем порогового значения $E_{t h}$ на $\mathrm{Z}+$ поверхности образовывались зародыши. Средняя плотность зародышей составляла $65 \mathrm{~mm}^{-2}$. При дальнейшем увеличении поля наблюдался медленный изотропный рост изолированных доменов (рис. 4). Следует отметить, что отжиг кристалла не влиял на скачкообразное движение плоских доменных стенок, образующихся вблизи краев электрода [25]. Однако в данном случае в местах остановки доменных стенок формировались „следы доменной стенки“ и остаточные домены [27]. При обратном переключении эти остаточные домены становились центрами зародышеобразования.

\section{6. Влияние высокотемпературного отжига на эволюцию доменной структуры}

Отжиг CLN при высокой температуре $\left(850^{\circ} \mathrm{C}\right)$ качественно изменял эволюцию доменной структуры. Анализ in situ оптических изображений позволил выделить

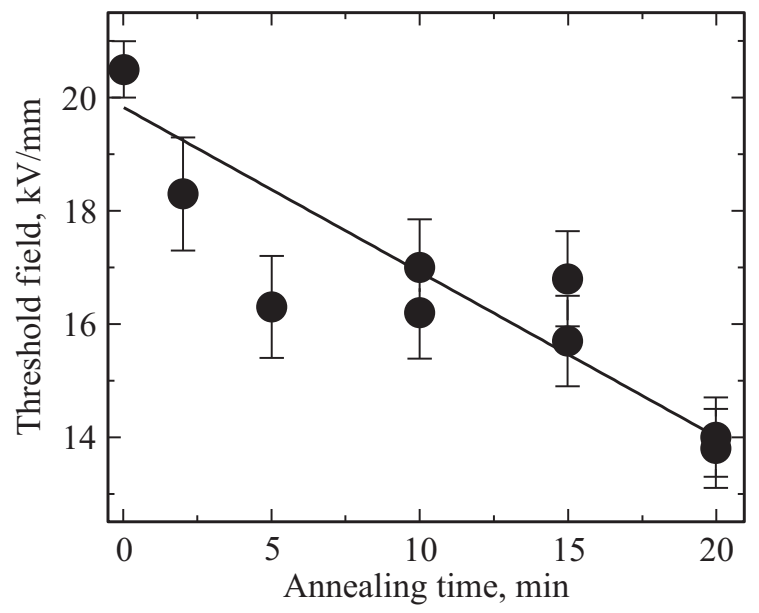

Рис. 3. Значения порогового поля переключения поляризации в кристаллах с различной длительностью отжига при температуре $850^{\circ} \mathrm{C}$.
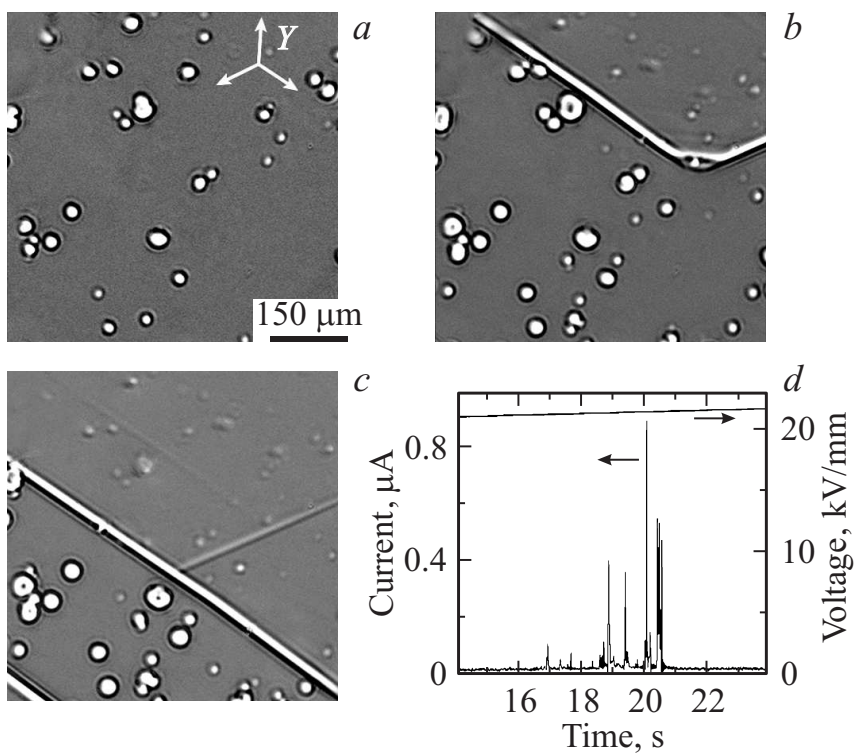

Рис. 4. Эволюция доменной структуры в CLN, отожженном при $300^{\circ} \mathrm{C}$ в течение $1 \mathrm{~h}$, Переключение в растущем поле: $(a)-21.3 \mathrm{kV} / \mathrm{mm} ;(b)-21.4 \mathrm{kV} / \mathrm{mm} ;(c)-21.5 \mathrm{kV} / \mathrm{mm}$. (d) - ток переключения.

два независимо протекающих процесса: (1) образование сетчатой доменной структуры под всей площадью, покрытой электродами и (2) плавное движение доменной границы. Сетчатая доменная структура формировалась одновременно на всей площади под электродом, а ее контраст усиливался при длительном приложении электрического поля. Такое поведение можно отнести за счет образования в процессе охлаждения после отжига приповерхностных зародышей, созданных пироэлектрическим полем (по аналогии со случаем низкотемпературного отжига).

Доменная граница перемещалась безостановочно от края к центру электрода. За счет изотропного роста 
граница сохраняла форму окружности (рис. 5, $a-c$ ). Плавное движение доменных стенок проявлялось в качественном изменении формы тока переключения в результате отжига (рис. 5,d). Выявлена линейная зависимость средней скорости движения доменной границы от приложенного поля (рис. 6).

Визуализация на боковом срезе статической доменной структуры после переключения поляризации при помощи оптического микроскопа после селективного химического травления показала, что доменная структура локализована преимущественно в объеме кристалла (рис. 7,a). При этом формируются две структуры с ЗДС - вблизи Z- поверхности (рис. $7, b)$ и в объеме (рис. $7, c)$ - с различными апертурами и периодами, со-
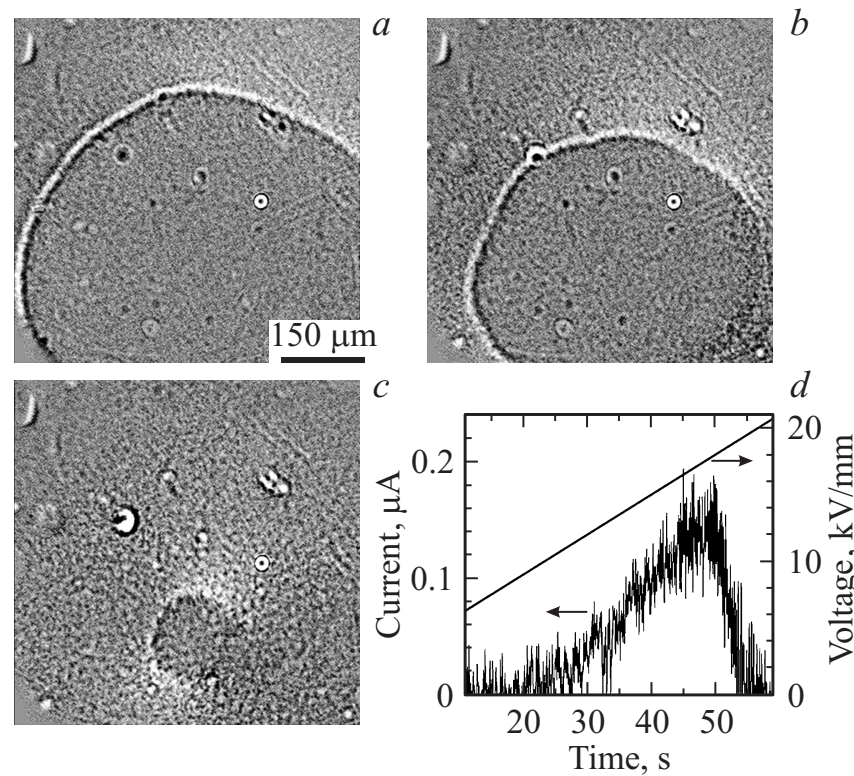

Pис. 5. Эволюция доменной структуры в CLN, отожженном при $850^{\circ} \mathrm{C}$ в течение $15 \mathrm{~min}$. Переключение в растущем поле: (a) $-18.1 \mathrm{kV} / \mathrm{mm} ;(b)-18.3 \mathrm{kV} / \mathrm{mm} ;$ (c) $-18.6 \mathrm{kV} / \mathrm{mm}$. $(d)$ - ток переключения.

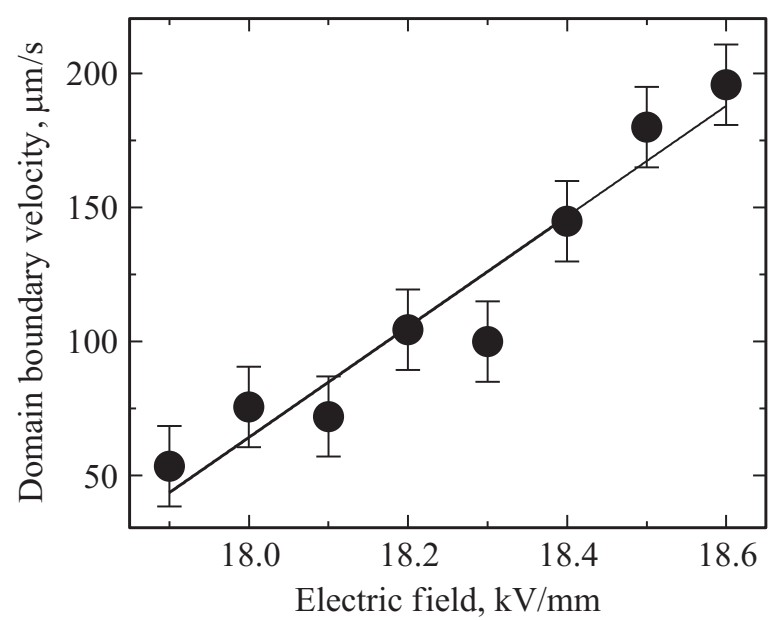

Рис. 6. Полевая зависимость средней скорости движения доменной границы в CLN, отожженном при $850^{\circ} \mathrm{C}$ в течение $15 \mathrm{~min}$.
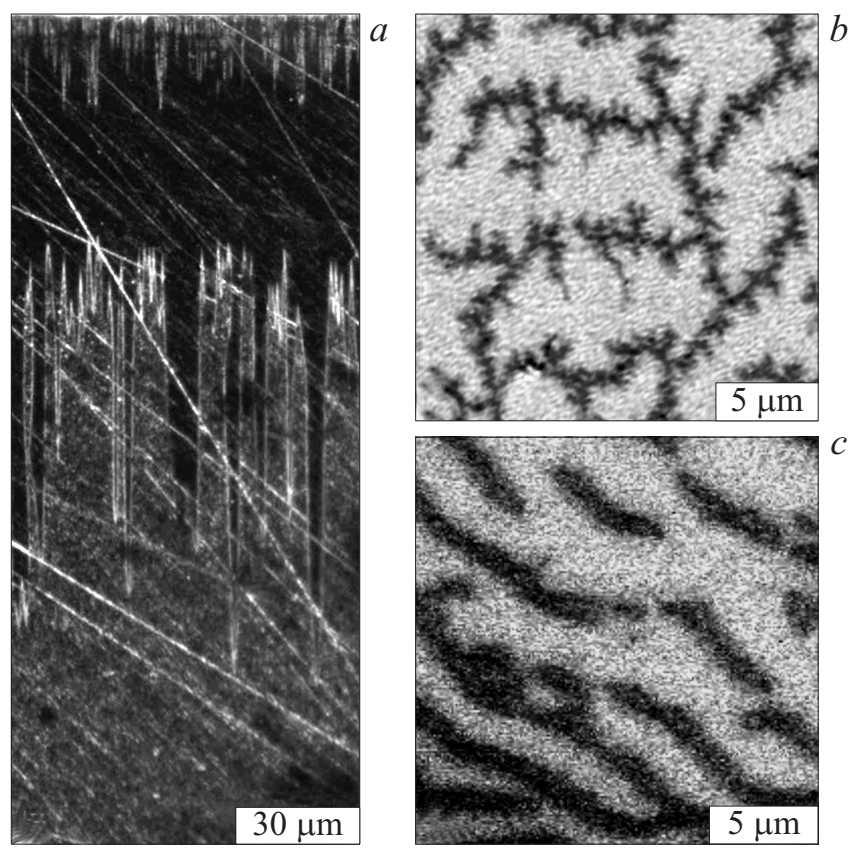

Рис. 7. Доменная структура: $(a)$ на боковом срезе - оптическая микроскопия после селективного химического травления, (b) на Z- поверхности - CMПО, (c) в объеме $(140 \mu \mathrm{m})-$ КМКР.

ставляющими соответственно $15 \pm 3 \mu \mathrm{m}$ и $1.5 \pm 0.2 \mu \mathrm{m}$ для Z- поверхности $70 \pm 15 \mu \mathrm{m}$ и $5 \pm 1.5 \mu \mathrm{m}$ для объема. Принципиально различалась и морфология ЗДС, которая представляла собой на Z- поверхности дендриты со средней шириной $850 \mathrm{~nm}$, а в объеме кристалла полосовые структуры со средней шириной около $2.2 \mu \mathrm{m}$.

\section{7. Обсуждение результатов}

Для описания наблюдаемой эволюции доменной структуры была предложена модель, учитывающая два механизма воздействия отжига в вакууме: (1) формирование нанодоменов и (2) неоднородное распределение электрического поля в объеме.

7.1. Формирование нанодоменов. Увеличение плотности зародышей в результате отжига при низкой температуре можно отнести за счет формирования нанодоменов (зародышей) под действием пироэлектрического поля, возникающего при охлаждении кристалла $[5,6]$. Образование сетчатой структуры на Z- поверхности в образцах после высокотемпературного отжига под всей площадью, покрытой электродом, также обусловлено существованием этих нанодоменов.

7.2. Неоднородное распределение электрического поля. Эволюция доменной структуры в образцах после высокотемпературного отжига существенно отличается от традиционного роста изолированных доменов в одноосных сегнетоэлектриках. Обычно, элементарные ступени образуются на доменных стенках на полярной поверхности кристалла и перемещаются 
вдоль доменных стенок в полярном направлении [25,27]. Неоднородное распределение электрического поля приводит к тому, что эффективная генерация ступеней наблюдается в объеме, поскольку вероятность генерации ступеней $P_{2 D}(E)$ (двумерного зародышеобразования) определяется локальным значением электрического поля

$$
P_{2 D}(E)=\exp \left[-E_{a c} /\left(E_{s}-E_{b}\right)\right]
$$

где $E_{a c}$ - поле активации, $E_{s}$ - локальное значение переключающего поля, $E_{b}$ - поле смещения.

При переключении в растущем поле удается реализовать такое распределение поля, при котором происходит только разрастание доменов в объеме вблизи максимума приложенного электрического поля. Локализованные в объеме домены не выходят на $\mathrm{Z}+$ поверхности, что приводит к формированию заряженных доменных стенок. Следует отметить, что увеличение проводимости в приповерхностном слое облегчает объемное экранирование деполяризующих полей, создаваемых ЗДС. Апертура и период ЗДС зависят от объемной проводимости и приложенного поля, что позволяет управлять геометрическими параметрами ЗДС.

Следует отметить, что метастабильная доменная структура с ЗДС весьма устойчива. В частности, отжигом в кислородной атмосфере модифицированных кристаллов с ЗДС можно восстановить исходную однородную электропроводность кристалла, сохраняя при этом ЗДС. Создание ЗДС с контролируемыми параметрами представляет значительный интерес для развития инженерии доменных границ.

\section{8. Заключение}

Экспериментально исследована эволюция доменной структуры при переключении поляризации в пластинах ниобата лития с пространственно неоднородной объемной проводимостью, созданной отжигом в вакууме. Установлено, что отжиг в вакууме приводит к (1) увеличению концентрации зародышей и (2) росту доменов в объеме с формированием заряженных доменных стенок. Формирование нанодоменов отнесено за счет переключения в пироэлектрическом поле, возникающем при охлаждении после отжига. Повышение проводимости в приповерхностном слое увеличивает вероятность зародышеобразования в объеме и облегчает объемное экранирование деполяризующих полей. Изменение условий отжига и параметров переключающего импульса может быть использовано для изменения геометрических параметров ЗДС. Создание ЗДС с контролируемыми параметрами представляет значительный интерес для инженерии доменных стенок.

Авторы выражают благодарность Палицыну И.С. за совместную работу.

\section{Список литературы}

[1] T. Volk, M. Wöhlecke. Lithium niobate: defects, photorefraction and ferroelectric switching. Springer Verlag, Berlin, Heidelberg. (2008). 250 p.

[2] Ю.С. Кузьминов. Электрооптический и нелинейно-оптический кристалл ниобата лития. Наука, М. (1987). 264 с.

[3] R.S. Weis, T.K. Gaylord. Appl. Phys. A 37, 191 (1985).

[4] V.Ya. Shur. Ferroelectrics 340, 3 (2006).

[5] V.Ya. Shur. In: Handbook of advanced dielectric, piezoelectric and ferroelectric materials. Synthesis, properties and applications. / Ed. Z.-G. Ye. Woodhead Publishing, Cambridge (2008). P. 622.

[6] V.Ya. Shur, A.R. Akhmatkhanov, I.S. Baturin. Appl. Phys. Rev. 2, 040604 (2015).

[7] V.Ya. Shur, E.L. Rumyantsev, E.V. Nikolaeva, E.I. Shishkin, R.G. Batchko, M.M. Fejer, R.L. Byer. Ferroelectrics 257, 191 (2001).

[8] E.A. Eliseev, A.N. Morozovska, G.S. Svechnikov, V. Gopalan, V.Ya. Shur. Phys. Rev. B 83, 235313 (2011).

[9] M. Schroder, A. Haussmann, A. Thiessen, E. Soergel, T. Woike, L.M. Eng. Adv. Funct. Mater. 22, 3936 (2012).

[10] S. Wada. Ferroelectrics 389, 3 (2009).

[11] V.Ya. Shur, P.S. Zelenovskiy, M.S. Nebogatikov, D.O. Alikin, M.F. Sarmanova, A.V. Ievlev, E.A. Mingaliev, D.K. Kuznetsov. J. Appl. Phys. 110, 052013 (2011).

[12] P.S. Zelenovskiy, M.D. Fontana, V.Ya. Shur, P. Bourson, D.K. Kuznetsov. Appl. Phys. A 99, 741 (2010).

[13] B.K. Brickeen, C. Shanta. Opt. Eng. 49, 124201 (2010).

[14] E. Perez-Enciso, S. Vieira, L. Arizmendi. Ferroelectrics 185, 17 (1996).

[15] A.Ye. Lushkin, V.B. Nazarenko, K.N. Pilipchak, V.F. Shnyukov, A.G. Naumovets. J. Phys. D. 32, 22 (1999).

[16] A. Dhar, N. Singh, R.K. Singh, R. Singh. J. Phys. Chem. Solids 74, 146 (2013).

[17] E. Schreck, K. Dransfeld. Appl. Phys. A 44, 265 (1987).

[18] J.L. Ketchum, K.L. Sweeney, L.E. Halliburton. Phys. Lett. A 94, 450 (1983).

[19] V.Ya. Shur, D.O. Alikin, A.V. Ievlev, M.A. Dolbilov, M.F. Sarmanova, N.V. Gavrilov. IEEE Trans. Ultrason. Ferroelectr. Freq. Control 59, 1934 (2012).

[20] A.L. Kholkin, S.V. Kalinin, A. Roelofs, A. Gruverman. In: Scanning probe microscopy / Ed. S. Kalinin, A. Gruverman. Springer, N.Y. (2007). P. 173.

[21] P.S. Zelenovskiy, M.D. Fontana, V.Ya. Shur, P. Bourson, D.K. Kuznetsov. Appl. Phys. A 99, 741 (2010).

[22] V.Ya. Shur, A.I. Lobov, A.G. Shur, S. Kurimura, Y. Nomura, K. Terabe, X.Y. Liu, K. Kitamura. Appl. Phys. Lett. 87, 022905 (2005).

[23] В.Я. Шур, А.Л. Груверман, Н.В. Коровина, М.З. Орлова, Л.В. Шерстобитова. ФТТ 30, 299 (1988).

[24] V.I. Pryakhina, D.O. Alikin, I.S. Palitsin, S.A. Negashev, V.Ya. Shur. Ferroelectrics 476, 109 (2015).

[25] V.Ya. Shur. In: Nucleation theory and applications. / Ed. J.W.P. Schmelzer, WILEY-VCH, Weinheim (2005). P. 178.

[26] V.I. Pryakhina, V.Ya. Shur, D.O. Alikin, S.A. Negashev. Ferroelectrics 439, 20 (2012).

[27] V.Ya. Shur. J. Mater. Sci. 41, 199 (2006). 